\title{
Gravitation waves from QCD and electroweak phase transitions
}

\author{
Yidian Chen, ${ }^{a, b}$ Mei Huang ${ }^{a, b, c}$ and Qi-Shu Yan $^{b}$ \\ ${ }^{a}$ Theoretical Physics Division, Institute of High Energy Physics, Chinese Academy of Sciences, \\ 19B Yuquan Road, Beijing 100049, P.R. China \\ ${ }^{b}$ School of Physical Sciences, University of Chinese Academy of Sciences, \\ 19A Yuquan Road, Beijing 100049, P.R. China \\ ${ }^{c}$ Theoretical Physics Center for Science Facilities, Chinese Academy of Sciences, \\ 19B Yuquan Road, Beijing 100049, P.R. China \\ E-mail: chenyd@ihep.ac.cn, huangm@ihep.ac.cn, yanqishu@ucas.ac.cn
}

ABSTRACT: We investigate the gravitation waves produced from QCD and electroweak phase transitions in the early universe by using a 5-dimension holographic QCD model and a holographic technicolor model. The dynamical holographic QCD model is to describe the pure gluon system, where a first order confinement-deconfinement phase transition can happen at the critical temperature around $250 \mathrm{MeV}$. The minimal holographic technicolor model is introduced to model the strong dynamics of electroweak, it can give a first order electroweak phase transition at the critical temperature around 100-360 GeV. We find that for both GW signals produced from QCD and EW phase transitions, in the peak frequency region, the dominant contribution comes from the sound waves, while away from the peak frequency region the contribution from the bubble collision is dominant. The peak frequency of gravitation wave determined by the QCD phase transition is located around $10^{-7} \mathrm{~Hz}$ which is within the detectability of FAST and SKA, and the peak frequency of gravitational wave predicted by EW phase transition is located at $0.002-0.007 \mathrm{~Hz}$, which might be detectable by BBO, DECIGO, LISA and ELISA.

Keywords: Gauge-gravity correspondence, Technicolor and Composite Models

ARXIV EPRINT: 1712.03470 


\section{Contents}

1 Introduction 1

2 First order confinement-deconfinement phase transition 3

2.1 Quenched dynamical holographic QCD model 3

2.2 First order Hawking-Page phase transition of confinement-deconfinement 4

3 The first order EW phase transition $\quad 6$

$\begin{array}{lll}3.1 & \text { The soft-wall holographic technicolor model } & 6\end{array}$

3.2 The first order Hawking-Page phase transition for EW 9

4 Gravitational wave $\quad 10$

5 Conclusions and discussions $\quad 15$

\section{Introduction}

Predicted by Albert Einstein on the basis of general relativity, gravitational radiation is generated by the changes of the curvature of spacetime and propagates outwards as a wave at the speed of light $[1,2]$. On February 11, 2016, the LIGO and Virgo Scientific Collaboration [3] announced the first observed gravitational waves (GWs) signal in the detectors of LIGO. The gravitational waves, named GW150914, were originated from a binary black hole merger. Recently, the gravitational waves, named GW170817, originated from a binary neutron star inspiral were observed in LIGO [4]. The Nobel Prize in Physics 2017 was awarded to Rainer Weiss, Kip Thorne and Barry Barish for their decisive contributions to the LIGO detector and the observation of gravitational waves, which opens a new exciting era for astronomy and cosmology.

The GWs can be roughly divided into three categories [5]: 1) GWs can be produced through various astrophysical processes, such as compact binary inspirals, explosion of supernova and spherically asymmetric spinning neutron stars, among them, binaries systems are the main sources for detecting GWs through ground-based detectors LIGO, Virgo and Space-based interferometers LISA, DECIGO, BBO. 2) The primordial GWs can be produced in the very early stages of the Universe, such as cosmic strings, the inflation and reheating epochs, and these primordial GWs have unique imprint on the cosmic microwave background; 3) GWs can be produced from cosmological phase transitions in the early universe, such as GUT, electroweak (EW) and Quantum chromodynamics (QCD) phase transition, and these GWs can tell us the evolution of the universe.

During the evolution of the universe, several phase transitions might have occurred. If it is of a first order phase transition, it can generate gravitational waves. When two 
local minima of a free energy coexist in a certain temperature range, the scalar field can enter into the broken phase from the symmetric phase via quantum tunnelling or thermally fluctuation, which can lead the nucleation of bubbles in the metastable sea. If this process is fast enough when compared to the rate of expansion Hubble parameter $H$, the bubbles will expand and collide with each other and produce the GWs [6-9].

When the universe cools down to around several hundreds GeV, the EW phase transition could happen. Although the phase transition in the electroweak sector of the Standard Model is crossover [10-12], first order phase transition is predicted in many extended scenarios beyond the Standard Model [13-37]. Therefore, the GW signals from the EW phase transition, which could be detected by LISA [8], can shed some lights on the new physics beyond the standard model. Moreover, the first order phase transition is also favoured in order to produce the observed baryon asymmetry [38, 39] via the electroweak baryogenesis mechanism. Therefore, gravitational waves physics has opened a new window for the research of the fundamental laws of particle physics and cosmology. In particular, it can serve as an important tool to study the dynamics origin of the EW symmetry breaking and new physics beyond the standard model. As one of the solutions to tackle the hierarchy problem, technicolor models was first introduced by Weinberg [40] and Susskind [41]. A more realistic technicolor model, like the walking technicolor [42-44] scenario, predicts a composite scalar boson techni-dilaton (TD) for a candidate of $125 \mathrm{GeV}$ boson. Similar to strong interaction of the QCD, the walking technicolor model can predict the EW phase transition and GWs generation in the evolution of the universe [45, 46].

When the universe further cools down to around several hundreds $\mathrm{MeV}$, the QCD phase transition happens, and the chiral symmetry is spontaneously broken and color degrees of freedom is confined. Exploring the QCD phase structure under extreme conditions is one the most important tasks for heavy ion collisions, especially in the Relativistic Heavy Ion Collisions (RHIC) and Large Hadron Collider (LHC), where two accelerated nucleus with relativistic velocities collide to create the hot quark-gluon plasma, which is normally called "little bang". Lattice QCD calculation shows that the phase transition for three light flavors is of smooth crossover at small baryon chemical potentia and high temperature [47, 48], and for heavy and static quarks or pure gauge theory, the QCD phase transition is of first order [49]. If the QCD phase transition is flavor dependent and happens sequentially [50], there might be chances for the appearance of first order QCD phase transition in the early universe. The GWs detection offers one more experimental tool to explore the QCD phase structure.

In order to tackle strongly coupled gauge theories (see [51-54] for review), the anti-de Sitter/conformal field theory (AdS/CFT) correspondence [55-57] or general gravity/gauge duality was proposed. In recent decades, many properties of QCD, for example, meson spectra [58-70], phase transitions and baryon number susceptibilities [71-75] have been investigated from both top-down and bottom-up models. Furthermore, new extensions beyond the standard models, such as technicolor models [76-85] and composite higgs models [86-90] have also been studied in the context of AdS/CFT.

In this work, by using a 5D dynamical holographic QCD model and a holographic technicolor model we investigate the first order phase transitions and predict the GW signals 
of these two models. The paper is organized as following: in section 2 we introduce the dynamical holographic QCD model for pure gluon system, and describe the first order deconfinement phase transition. Similar to holographic QCD model, in section 3 we construct the five dimensional holographic technicolor model, where a first order phase transition for EW can be described. We calculate the GWs from EW and QCD phase transitions in section 4 . Finally, a short summary is given in section 5 .

\section{First order confinement-deconfinement phase transition}

\subsection{Quenched dynamical holographic QCD model}

In order to tackle the challenging from strong coupling in the infrared (IR) of QCD, in recent decades, the anti-de Sitter/conformal field theory correspondence or the gauge/gravity duality [55-57] has been widely applied in investigating hadron physics, strongly coupled quark gluon plasma, QCD phase transitions and transport properties. It can be regarded as an general principle that for any d-dimensional quantum field theory (QFT) there exists a dual theory of quantum gravity living in $(\mathrm{d}+1)$-dimensions, and the gravitational description becomes classical when the QFT is strongly-coupled. Here the extra dimension, i.e., the 5th-dimension can be also interpreted as an energy scale or renormalization group (RG) flow in the QFT [91].

In the past decade, much effort has been paid from both top-down and bottom-up methods on constructing a realistic holographic QCD model. From bottom-up, the most economic way of breaking the conformal symmetry is to add a proper deformed warp factor in front of the $\mathrm{AdS}_{5}$ metric, which can capture the main non-perturbative QCD features. For example, a quadratic correction in front of the warp factor of $\mathrm{AdS}_{5}$ geometry [92] or a deformed warp factor which mimics the QCD running coupling [93] can help to realize the linear heavy quark potential. For the hadron spectra, based on the hard-wall AdS/QCD model [94] and the soft-wall AdS/QCD or KKSS model [60], much effort has been made [95-99] to realize the spontaneously chiral symmetry breaking and linear confinement properties in hadron spectra. A dynamical holographic QCD (DhQCD) model has been developed in the systematic graviton-dilaton-scalar framework [99], with the dilaton background field $\Phi(z)$ and the scalar field $X(z)$ describing nonperturbative gluodynamics and chiral dynamics, respectively. The metric structure at IR in the DhQCD model can be automatically deformed by the nonperturbative gluon condensation and chiral condensation in the vacuum, and the model is quite successful in describing hadron spectra [100], QCD equation of state [101], QCD phase transitions and transport properties [102, 103].

Here in this work, we only focus on the pure gluon system, which can be described by the quenched dynamical holographic QCD model in the graviton-dilaton framework. The action in the string frame takes the form of:

$$
S_{G}=\frac{1}{16 \pi G_{5}} \int d^{5} x \sqrt{g_{s}} e^{-2 \Phi}\left(R_{s}+4 \partial_{M} \Phi \partial^{M} \Phi-V_{G}^{s}(\Phi)\right) .
$$

Here $G_{5}$ is the $5 \mathrm{D}$ Newton constant, and $g_{s}, \Phi(z)$ and $V_{G}^{s}$ are the $5 \mathrm{D}$ metric, the dilaton field and dilaton potential in the string frame, respectively. The dilaton field takes the 
quadratic form of $\Phi(z)=\mu_{G}^{2} z^{2}$ to produce linear confinement at IR [60]. This quenched DhQCD model can describe well not only the scalar glueball spectra [100], but also all two-gluon and three-gluon glueballs including vectors and tensors [104].

\subsection{First order Hawking-Page phase transition of confinement-deconfinement}

The gauge theory at finite temperature has a holographic counterpart in the thermodynamics of black-holes on the gravity side. Adding the black-hole background to the quenched dynamical holographic QCD model constructed from vacuum properties, the metric in the string frame takes the form of

$$
d s_{S}^{2}=e^{2 A_{s}}\left(-f(z) d t^{2}+\frac{d z^{2}}{f(z)}+d x^{i} d x^{i}\right) .
$$

However, the thermodynamical properties including phase transitions and equation of state are convenient to be derived in the Einstein frame, which is described by

$$
d s_{E}^{2}=e^{2 A_{s}-\frac{4 \Phi}{3}}\left(-f(z) d t^{2}+\frac{d z^{2}}{f(z)}+d x^{i} d x^{i}\right) .
$$

Under the transformation between the string fame and the Einstein frame as following:

$$
g_{m n}^{E}=g_{m n}^{s} e^{-2 \Phi / 3}, \quad V_{G}^{E}=e^{4 \Phi / 3} V_{G}^{s},
$$

the action at string frame eq. (2.1) becomes

$$
S_{G}^{E}=\frac{1}{16 \pi G_{5}} \int d^{5} x \sqrt{g_{E}}\left(R_{E}-\frac{4}{3} \partial_{m} \Phi \partial^{m} \Phi-V_{G}^{E}(\Phi)\right)
$$

in the Einstein frame.

One can derive the following equations of motion (EOMs):

$$
\begin{aligned}
-A_{s}^{\prime \prime}+A_{s}^{\prime 2}+\frac{2}{3} \Phi^{\prime \prime}-\frac{4}{3} A_{s}^{\prime} \Phi^{\prime} & =0, \\
f^{\prime \prime}(z)+\left(3 A_{s}^{\prime}(z)-2 \Phi^{\prime}(z)\right) f^{\prime}(z) & =0, \\
\frac{8}{3} \partial_{z}\left(e^{3 A_{s}(z)-2 \Phi} f(z) \partial_{z} \Phi\right)-e^{5 A_{s}(z)-\frac{10}{3} \Phi} \partial_{\Phi} V_{G}^{E} & =0,
\end{aligned}
$$

and the solution of the black-hole background takes the form of

$$
f(z)=1-f_{c}^{h} \int_{0}^{z} e^{-3 A_{s}\left(z^{\prime}\right)+2 \Phi\left(z^{\prime}\right)} d z^{\prime},
$$

with

$$
f_{c}^{h}=\frac{1}{\int_{0}^{z_{h}} e^{-3 A_{s}\left(z^{\prime}\right)+2 \Phi\left(z^{\prime}\right)} d z^{\prime}} .
$$

We have $f\left(z_{h}\right)=0$ at the horizon $z=z_{h}$. The periodicity of the Euclidean time

$$
\tau \rightarrow \tau+\frac{4 \pi}{\left|f^{\prime}\left(z_{h}\right)\right|}
$$




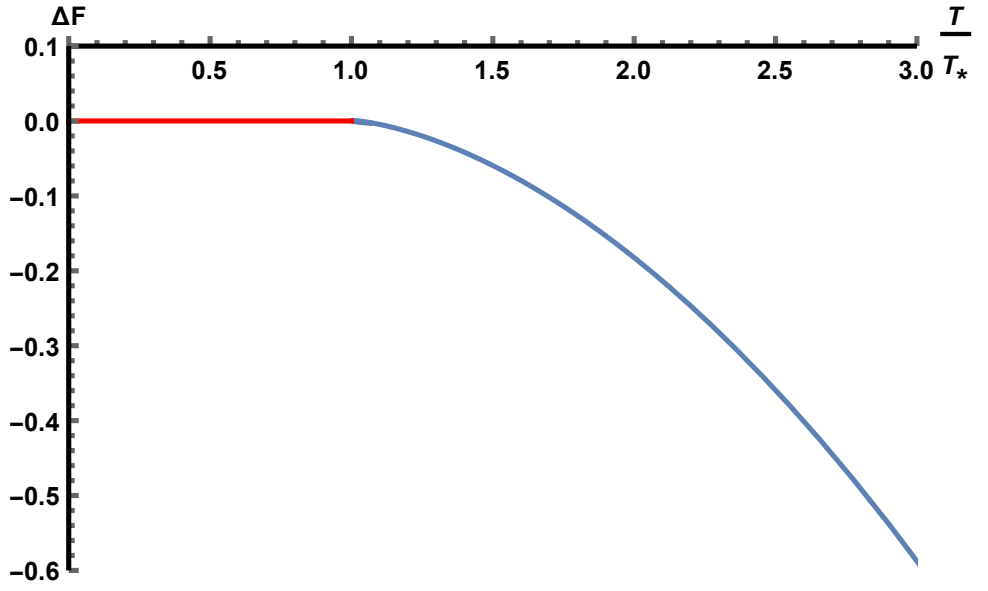

Figure 1. The free energy difference of the quenched dynamical holographic QCD model as a function of $T / T_{*}$. The parameters $\mu_{G}=0.75 \mathrm{GeV}$ and $G_{5}=1.25$ are used and the critical temperature is $T_{*}=255 \mathrm{MeV}$.

determines the temperature of the solution as

$$
T=\frac{\left|f^{\prime}\left(z_{h}\right)\right|}{4 \pi},
$$

then one can easily find the relation between the temperature and position of the black hole horizon,

$$
T=\frac{e^{-3 A_{s}\left(z_{h}\right)+2 \Phi\left(z_{h}\right)}}{4 \pi \int_{0}^{z_{h}} e^{-3 A_{s}\left(z^{\prime}\right)+2 \Phi\left(z^{\prime}\right)} d z^{\prime}} .
$$

With the parameters $\mu_{G}=0.75 \mathrm{GeV}$ and $G_{5}=1.25$ used in [102], we can get the critical temperature $T_{*}=255 \mathrm{MeV}$ for the first order Hawking-Page confinement-deconfinement phase transition. The first order phase transition behavior can be also read from the free energy difference $\Delta F=F^{B H}-F_{T G}$ as a function of the temperature shown in figure 1 . Above the critical temperature $T>T_{*}$, by using the saddle point approximation, the free energy density for the black-hole has the form of

$$
F^{B H} \simeq T S^{B H}=\frac{T}{\kappa_{5}^{2}} \int_{0}^{T^{-1}} d t \int_{\epsilon}^{z_{h}} d z e^{5 A(z)-2 \Phi(z)} \frac{2}{3} V(\phi)^{B H},
$$

and below the critical temperature $T<T_{*}$, the free energy density for the thermal gas takes the form of

$$
F_{T G} \simeq T S_{T G}=\frac{T}{\kappa_{5}^{2}} \int_{0}^{\beta^{\prime}} d t \int_{\epsilon}^{z_{I R}} d z e^{5 A(z)-2 \Phi(z)} \frac{2}{3} V(\phi)_{T G}
$$

As we can see that the free energy difference $\Delta F$ keeps zero below the critical temperature $T<T_{*}$, and in the region above the critical temperature $T>T_{*}$, the free energy difference decreases monotonically with the temperature, which is a typical behavior for first order phase transition. 


\section{The first order EW phase transition}

\subsection{The soft-wall holographic technicolor model}

In the SM, the electroweak phase transition is a crossover [10-12]. However, when the technicolor models or more general strongly coupled scenarios are considered, (strongly) first-order phase transition at the electroweak or $\mathrm{TeV}$ scale is possible. We apply the gauge/gravity duality to model the strong dynamics of electroweak on five dimensional (5D) anti-de Sitter spacetime $\left(\mathrm{AdS}_{5}\right)$. Similar to the bottom-up holographic model of QCD [58, 59], we construct a 5D holographic technicolor model or a phenomenological " $D p-D q$ " model with the total action of

$$
S_{T C}=S_{G}+S_{5} .
$$

$S_{G}$ is the background " $D p$ " brane action

$$
S_{G}=\frac{1}{2 \kappa_{5}^{2}} \int d^{5} x \sqrt{g} e^{-w(z)}\left(R+\frac{12}{L^{2}}\right),
$$

and the background is described by the anti-de Sitter spacetime $\left(\mathrm{AdS}_{5}\right)$ metric

$$
d s^{2}=g_{M N} d x^{M} d x^{N}=(L / z)^{2}\left(\eta_{\mu \nu} d x^{\mu} d x^{\nu}+d z^{2}\right),
$$

with $R$ the Ricci scalar, $L$ the curvature radius of $\mathrm{AdS}_{5}$ and the negative cosmological constant $-12 / L^{2}$. The probe flavor " $D q$ " brane with $\mathrm{SU}\left(N_{\mathrm{TF}}\right)_{L} \times \mathrm{SU}\left(N_{\mathrm{TF}}\right)_{R}$ gauge symmetry ( $N_{\mathrm{TF}}$ the number of techni-flavors) living on the $\left(\mathrm{AdS}_{5}\right)$ background is described by the action $S_{5}$, which takes the form of

$$
\begin{aligned}
S_{5}=-\int d^{4} x \int_{\epsilon}^{z_{m}} d z \sqrt{-g} e^{-w(z)} \operatorname{Tr}( & \left|\left(D^{M} U\right)^{\dagger}\left(D_{M} U\right)\right|+m_{5}^{2}\left|U^{\dagger} U\right| \\
& \left.+\frac{1}{4 g_{5}^{2}}\left(L^{M N} L_{M N}+R^{M N} R_{M N}\right)\right) .
\end{aligned}
$$

Where the covariant derivative is defined as $D_{M} U=\partial_{M} U-i L_{M} U+i U R_{M}, L(R)_{M N}$ is defined as $L(R)_{M N}=\partial_{M} L(R)_{N}-\partial_{N} L(R)_{M}-i\left[L(R)_{M}, L(R)_{N}\right]$, and the gauge coupling $g_{5}$ is fixed by the UV asymptotic forms of the vector current two-point function as $g_{5}^{2}=$ $12 \pi^{2} L / N_{\mathrm{TC}}[58,76,77]$ with $N_{\mathrm{TC}}$ the number of colors. The fifth coordinate is restricted from the infrared (IR) cut-off $z_{m}$ to the ultraviolet (UV) cut-off $\epsilon$, and we always imply the limit of $\epsilon \rightarrow 0$ for simplicity. In eqs. (3.2) and (3.4), we have introduced a soft-wall $w(z)$ to break the scale symmetry breaking. It should be noticed that here $w(z)$ is just a soft cut-off not a dynamical field, therefore, there is no corresponding potential in eq. (3.2) thus $w(z)$ is not needed to be solved from the Einstein equation.

The bulk scalar field $U$ is dual to the boundary operator $\left\langle\bar{Q}_{\mathrm{TC}} Q_{\mathrm{TC}}\right\rangle$ the chiral flavor symmetry in the boundary theory corresponds to left- and right-gauge fields in the bulk. The $5 \mathrm{D}$ mass parameter is related to $m_{5}^{2}=-\left(3-\gamma_{m}\right)\left(1+\gamma_{m}\right) / L^{2}$, and $\gamma_{m} \simeq 1$ is the same setup as $[76,77]$. The bulk scalar fields $U$ can be decomposed as

$$
U=\frac{1}{\sqrt{2 N_{\mathrm{TF}}}}(v(z)+\sigma(x, z)) e^{2 i \Pi^{a}(x, z) t^{a}} .
$$


Expanding (3.4), we find the coupled equations of motion for the vacuum expectation values $v(z)$,

$$
-\frac{1}{e^{-w(z)}} \partial_{z}\left(\frac{L^{3}}{z^{3}} e^{-w(z)} \partial_{z} v(z)\right)+\frac{L^{5}}{z^{5}} m_{5}^{2} v(z)=0 .
$$

We choose the UV boundary condition for $v(z)$ as

$$
\left.v(z)\right|_{z \rightarrow \epsilon}=M z^{2}
$$

where $M$ stands for the current mass of techni-quarks.

Next we expand all the fields in terms of Kaluza-Klein(KK) modes. The Higgs boson in standard model corresponds to the lowest KK mode of bulk scalar $U$ in technicolor scenario named techni-dilaton. Expanding the scalar field $\sigma(x, z)=\sum_{n} f_{\sigma}^{(n)}(x) \sigma^{(n)}(z)$, the equations of motion for the $\sigma_{n}(z)$ is,

$$
-\frac{z^{3}}{L^{3} e^{-w(z)}} \partial_{z}\left(\frac{L^{3} e^{-w(z)}}{z^{3}} \partial_{z} \sigma_{n}\right)+\frac{L^{2}}{z^{2}} m_{5}^{2} \sigma_{n}=m_{n}^{2} \sigma_{n} .
$$

Similar to the [58], we choose their boundary conditions as

$$
\partial_{z} \sigma_{n}\left(z_{m}\right)=0, \quad \sigma_{n}(\epsilon)=0 .
$$

We introduce the vector and axial-vector fields as $V_{M}=\left(L_{M}+R_{M}\right) / 2$ and $A_{M}=\left(L_{M}-\right.$ $\left.R_{M}\right) / 2$. In $V_{z}=A_{z}=0$ gauge, the equations of motion for the transverse part of the gauge field are

$$
\begin{array}{r}
-\frac{z}{e^{-w(z)} L} \partial_{z}\left(\frac{e^{-w(z)} L}{z} \partial_{z} V_{n}^{a}\right)=m_{n}^{2} V_{n}^{a}, \\
-\frac{z}{e^{-w(z)} L} \partial_{z}\left(\frac{e^{-w(z)} L}{z} \partial_{z} A_{n}^{a}\right)+\frac{v^{2} g^{2} L^{2}}{z^{2}} A_{n}^{a}=m_{n}^{2} A_{n}^{a} .
\end{array}
$$

The boundary conditions are chosen as:

$$
\begin{aligned}
\partial_{z} V_{n}^{a}\left(z_{m}\right) & =\partial_{z} A_{n}^{a}\left(z_{m}\right)=0 \\
V_{n}^{a}(\epsilon) & =0, \quad A_{n}^{a}(\epsilon)=0 .
\end{aligned}
$$

It is similar to the holographic QCD case that we can get a set of vector and axialvector mesons which the lowest eigenvalue is identified as the techni- $\rho$ and techni- $a_{1}$ meson, respectively. The pseudo-scalar field is coupled with longitudinal part of axial-vector field. The resulting equations of motion $\operatorname{are}\left(A_{\mu}=A_{\mu \perp}+\partial_{\mu} \varphi\right)$

$$
\begin{aligned}
& \partial_{z}\left(\frac{e^{-w(z)} L}{z} \partial_{z} \varphi_{n}^{a}\right)+\frac{g_{5}^{2} v^{2} e^{-w(z)} L^{3}}{z^{3}}\left(\Pi_{n}^{a}-\varphi_{n}^{a}\right)=0 \\
&-m_{n}^{2} \partial_{z} \varphi_{n}^{a}+\frac{g_{5}^{2} v^{2} e^{-w(z)} L^{2}}{z^{2}} \partial_{z} \Pi_{n}^{a}=0 .
\end{aligned}
$$

We choose their boundary conditions as:

$$
\partial_{z} \varphi_{n}^{a}\left(z_{m}\right)=\varphi_{n}^{a}(\epsilon)=\Pi_{n}^{a}(\epsilon)=0
$$




\begin{tabular}{|c|c|c|c|c|c|c|c|}
\hline & \multicolumn{3}{|c|}{ Model I } & \multicolumn{3}{|c|}{ Model II } \\
\hline & & $N_{\mathrm{TC}}=3$ & $N_{\mathrm{TC}}=4$ & $N_{\mathrm{TC}}=5$ & $N_{\mathrm{TC}}=3$ & $N_{\mathrm{TC}}=4$ & $N_{\mathrm{TC}}=5$ \\
\hline \multirow{3}{*}{$\begin{array}{l}\vec{\Xi} \\
\stackrel{2}{\Xi} \\
\vec{\Xi}\end{array}$} & $z_{m}^{-1}(\mathrm{TeV})$ & 1.072 & 1.079 & 1.083 & 0.299 & 0.299 & 0.299 \\
\hline & $c$ & 1.395 & 1.414 & 1.423 & 0.099 & 0.099 & 0.099 \\
\hline & $M$ & 0.553 & 0.541 & 0.534 & 0.380 & 0.329 & 0.295 \\
\hline \multirow{8}{*}{ 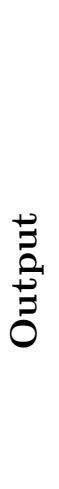 } & $S$ & \multicolumn{3}{|c|}{0.15 (fixed) } & 0.721 & 0.888 & 1.034 \\
\hline & $T_{*}(\mathrm{GeV})$ & 356 & 358 & 360 & \multicolumn{3}{|c|}{ 100(fixed) } \\
\hline & $m_{\text {higgs }}^{(1)}$ & 4.368 & 4.397 & 4.413 & 1.217 & 1.217 & 1.217 \\
\hline & $m_{\text {techni- } \rho}^{(0)}$ & 2.054 & 2.068 & 2.075 & 0.586 & 0.586 & 0.586 \\
\hline & $m_{\text {techni- } \rho}^{(1)}$ & 5.722 & 5.759 & 5.780 & 1.603 & 1.603 & 1.603 \\
\hline & $m_{\text {techni- }-a_{1}}^{(0)}$ & 2.342 & 2.274 & 2.236 & 2.853 & 2.292 & 1.882 \\
\hline & $m_{\text {techni- } a_{1}}^{(1)}$ & 5.814 & 5.824 & 5.830 & 3.090 & 2.515 & 2.242 \\
\hline & $m_{\Pi}^{(1)}$ & 5.531 & 5.521 & 5.515 & 3.135 & 2.686 & 2.385 \\
\hline
\end{tabular}

Table 1. Model I fits three experimental data $S=0.15, m_{\text {higgs }}^{(0)}=125 \mathrm{GeV}$ and $f_{\Pi}=246 \mathrm{GeV}$. Model II fits two experimental data $m_{\text {higgs }}^{(0)}=125 \mathrm{GeV}, f_{\Pi}=246 \mathrm{GeV}$ and assumes a phase transition temperature of $100 \mathrm{GeV}$. Note that the unit of particle mass is $\mathrm{TeV}$.

The techni-pion decay constant and $S$ parameter is given as $[76,77]$

$$
\begin{aligned}
f_{\Pi}^{2} & =-\left.\frac{1}{g_{5}^{2}} \frac{L}{z} e^{-w(z)} \partial_{z} A(0, z)\right|_{z=\epsilon}, \\
S & =N_{D} 4 \pi \frac{L}{g_{5}^{2}} \int_{\epsilon}^{z_{m}} d z \frac{1}{z} e^{-w(z)}(1-A(0, z)) .
\end{aligned}
$$

Where $N_{D}$ is the number of generation.

Similar to the soft-wall holographic QCD model in ref. [60], we assume the soft-wall has the quadratic form $w(z)=-c z^{2}$. There are six free parameters $N_{\mathrm{TF}}, N_{\mathrm{TC}}, N_{D}, z_{m}$, $M$ and $c$ in our model. For simplicity, we fix the $N_{\mathrm{TF}}=2$ and $N_{D}=1$ as the minimal technicolor model, then only four free parameters are left. In order to produce realistic mass spectra, we choose the Higgs boson mass, techni-pion decay constant and the $S$ parameter as input to trade off with the parameters $\left(z_{m}, M, c\right)$. Then once the value of $N_{\mathrm{TC}}$ is fixed, all spectra can be found. According to the PDG [105], the electroweak precision tests put a constraint to the $\mathrm{S}$ parameter as $-0.01 \leq S \leq 0.15$ (at 90\% CL) assuming another oblique parameter $U=0$. The technicolor scenario requires the techni-pion decay constant $f_{\Pi}=246 \mathrm{GeV}$. As shown in table 1, for Model I, by fitting to three experimental data $S=0.15, m_{\text {higgs }}=125 \mathrm{GeV}$ and $f_{\Pi}=246 \mathrm{GeV}$, three free parameters $\left(z_{m}, M, c\right)$ can be found with a fixed value of $N_{\mathrm{TC}}=3,4,5$, respectively. On this basis, we obtained the techni-mesons spectra for $N_{\mathrm{TC}}=3,4$ and 5 , respectively. We find that in by using the parameters set of Model I all the masses of techni-mesons are heavier than $2 \mathrm{TeV}$. 


\subsection{The first order Hawking-Page phase transition for EW}

We have assumed that the flavor brane is a probe, therefore the thermodynamical properties of the system is dominated by the background action $S_{G}$ in eq. (3.2). The thermal AdS solution in Poincaré patch is

$$
d s^{2}=\frac{L^{2}}{z^{2}}\left(d t^{2}+d \vec{x}^{2}+d z^{2}\right), \quad \epsilon \leq z \leq z_{m} .
$$

The temperature of thermal AdS is $T=1 / \beta^{\prime}$, where $\beta^{\prime}$ is the period of the Euclidean time. The AdS-BH solution is

$$
d s^{2}=\frac{L^{2}}{z^{2}}\left(f(z) d t^{2}+d \vec{x}^{2}+\frac{d z^{2}}{f(z)}\right), \quad \epsilon \leq z \leq \min \left(z_{m}, z_{h}\right),
$$

with $f(z)=1-z^{4} / z_{h}^{4}$. The Hawking temperature of the black hole is $T=1 /\left(\pi z_{h}\right)$.

By choosing the soft-wall in the form of $w(z)=-c z^{2}$, we can compute the free energy for thermal AdS and AdS black hole

$$
\begin{aligned}
F^{\mathrm{AdS}} \simeq & \frac{4 L^{3} T}{\kappa_{5}^{2}} \int_{0}^{\beta^{\prime}} d t \int_{\epsilon}^{z_{m}} d z z^{-5} e^{c z^{2}} \\
= & \frac{L^{3}}{\kappa_{5}^{2}}\left[-\frac{e^{c z_{m}^{2}}}{z_{m}^{4}}-\frac{c e^{c z_{m}^{2}}}{z_{m}^{2}}+\frac{e^{c z_{m}^{2}}}{\epsilon^{4}}+\frac{c e^{c z_{m}^{2}}}{\epsilon^{2}}+c^{2}\left(\operatorname{Ei}\left(c z_{m}^{2}\right)-\operatorname{Ei}\left(c \epsilon^{2}\right)\right)\right] \\
F^{B H} \simeq & \frac{4 L^{3} T}{\kappa_{5}^{2}} \int_{0}^{\pi z_{h}} d t \int_{\epsilon}^{\min \left(z_{m}, z_{h}\right)} d z z^{-5} e^{c z^{2}} \\
= & \frac{L^{3}}{\kappa_{5}^{2}}\left[-\frac{e^{c\left(\min \left(z_{m}, z_{h}\right)\right)^{2}}}{\left(\min \left(z_{m}, z_{h}\right)\right)^{4}}-\frac{c e^{c\left(\min \left(z_{m}, z_{h}\right)\right)^{2}}}{\left(\min \left(z_{m}, z_{h}\right)\right)^{2}}\right. \\
& \left.\quad+\frac{e^{c\left(\min \left(z_{m}, z_{h}\right)\right)^{2}}}{\epsilon^{4}}+\frac{c e^{c\left(\min \left(z_{m}, z_{h}\right)\right)^{2}}}{\epsilon^{2}}+c^{2}\left(\operatorname{Ei}\left(c\left(\min \left(z_{m}, z_{h}\right)\right)^{2}\right)-\operatorname{Ei}\left(c \epsilon^{2}\right)\right)\right]
\end{aligned}
$$

respectively. The free energy difference is

$$
\Delta F= \begin{cases}\frac{L^{3}}{\kappa_{5}^{2}} \frac{1}{2 z_{h}^{4}} & z_{m}<z_{h} \\ \frac{L^{3}}{\kappa_{5}^{2}}\left[\frac{1-2 e^{c z_{h}^{2}\left(1+c z_{h}^{2}\right)}}{2 z_{h}^{4}}+\frac{e^{c z_{m}^{2}}\left(1+c z_{m}^{2}\right)}{z_{m}^{4}}+c^{2}\left(\operatorname{Ei}\left(c z_{h}^{2}\right)-\operatorname{Ei}\left(c z_{m}^{2}\right)\right)\right] & z_{m}>z_{h},\end{cases}
$$

where $\operatorname{Ei}(\mathrm{x}) \equiv \int_{-x}^{\infty} d t e^{-t} / t$.

The 1st-order Hawking-Page phase transition occurs when free energy difference changes the sign as shown in figure 2. For Model I in table 1, the EW phase transition happens at the critical temperature $T_{*}=356,358,360 \mathrm{GeV}$ for $N_{T C}=3,4,5$, respectively. Considering the typical critical temperature for EW phase transition is around $80-150 \mathrm{GeV}$, by fixing the critical temperature at $T_{*}=100 \mathrm{GeV}$, and fitting $m_{\text {higgs }}^{(0)}=125 \mathrm{GeV}, f_{\Pi}=246 \mathrm{GeV}$, we can have a rather large $S$ parameter $0.721,0.888,1.034$ for $N_{T C}=3,4,5$, respectively, which can be read from Model II of table 1. On this basis, we obtained the techni-mesons spectra for $N_{\mathrm{TC}}=3,4$ and 5 , respectively. We find that comparing with Model I, the masses of techni-mesons in Model II are much lighter. 

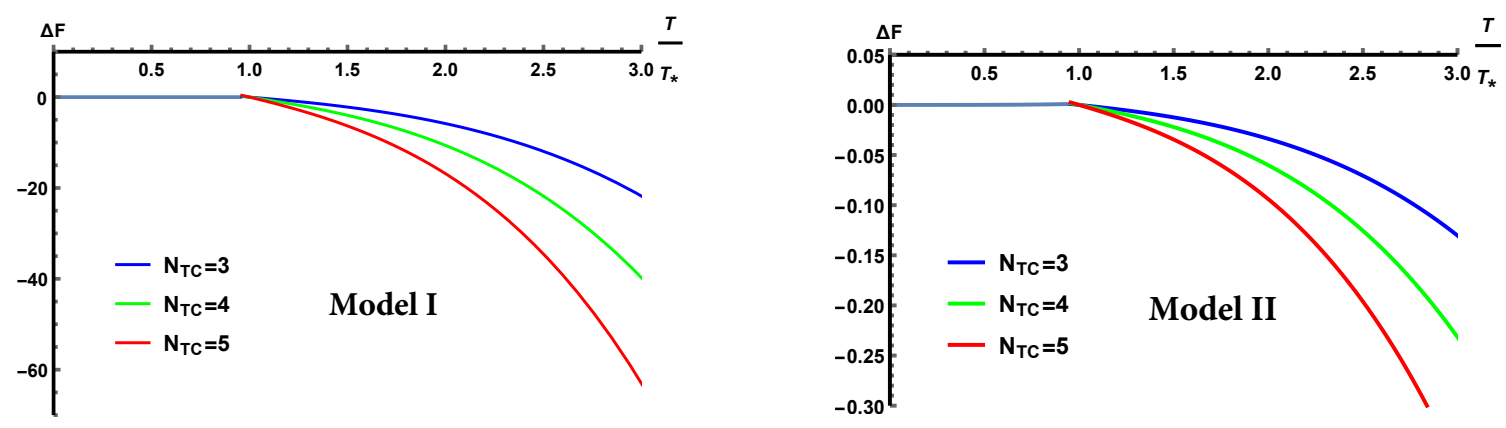

Figure 2. The free energy difference for Model-I and Model-II with $N_{\mathrm{TC}}=3, N_{\mathrm{TC}}=4$ and $N_{\text {TC }}=5$, respectively.

\section{Gravitational wave}

When the first-order phase transition takes place, the bubbles can nucleate in the supercooling plasma and the latent heat of free energy between symmetric and broken phases can engine the expansion of the bubble walls. Gravitational waves can be generated by the bubbles collisions [106, 107], by the stirred acoustic waves [108, 109] and by the Magnetohydrodynamic(MHD) turbulence in the plasma $[110,111]$ (see $[8,112]$ for review).

The gravitational waves power spectra are consisted of these three components, which can be put as

$$
h^{2} \Omega(f)=h^{2} \Omega_{\mathrm{env}}(f)+h^{2} \Omega_{\mathrm{sw}}(f)+h^{2} \Omega_{\mathrm{turb}}(f) .
$$

The gravitational wave power spectrum from bubble collisions in the envelope approximation can be given as $h^{2} \Omega_{\mathrm{env}}(f)$, which takes the following form [106] via numerical calculation

$$
h^{2} \Omega_{\mathrm{env}}(f)=1.67 \times 10^{-5} \Delta\left(\frac{H_{*}}{\beta}\right)^{2}\left(\frac{\kappa_{\phi} \alpha}{1+\alpha}\right)^{2}\left(\frac{100}{g_{*}}\right)^{\frac{1}{3}} S_{\mathrm{env}}(f),
$$

where the spectral form $S_{\text {env }}(f)$ have the power law which is given as

$$
S_{\text {env }}(f)=\frac{3.8\left(f / f_{\text {env }}\right)^{2.8}}{1+2.8\left(f / f_{\text {env }}\right)^{3.8}}
$$

Here $\alpha$ is the ratio of vacuum energy density over radiation energy density, $g_{*}$ is the number of active degrees of freedom during the phase transition, $v_{\mathrm{w}}$ is the velocity of bubble wall expansion, $\kappa_{\phi}$ is the coefficient which measure the efficiency of converting vacuum energy into scalar field gradient energy and $H_{*} / \beta$ is the nucleation rate relative to the Hubble rate during the phase transition which measures the time duration of phase transition.

The peak frequency of the GW produced by bubble collisions can be estimated as

$$
f_{\mathrm{env}}=16.5 \mu \mathrm{Hz}\left(\frac{f_{*}}{\beta}\right)\left(\frac{\beta}{H_{*}}\right)\left(\frac{T_{*}}{100 \mathrm{GeV}}\right)\left(\frac{g_{*}}{100}\right)^{\frac{1}{6}},
$$

where $T_{*}$ denotes the temperature during the phase transition. The dependence of the amplitude and peak frequency on the velocity of bubble expansion $v_{\mathrm{w}}$ is provided as

$$
\Delta=\frac{0.11 v_{\mathrm{w}}^{3}}{0.42+v_{\mathrm{w}}^{2}} ; \quad \frac{f_{*}}{\beta}=\frac{0.62}{1.8-0.1 v_{\mathrm{w}}+v_{\mathrm{w}}^{2}} .
$$


The power spectrum of GW from acoustic waves can be found in reference [108], which can take the following form

$$
h^{2} \Omega_{\mathrm{sw}}(f)=2.65 \times 10^{-6} v_{\mathrm{w}}\left(\frac{H_{*}}{\beta}\right)\left(\frac{\kappa_{\mathrm{f}} \alpha}{1+\alpha}\right)^{2}\left(\frac{100}{g_{*}}\right)^{\frac{1}{3}} S_{\mathrm{sw}}(f)
$$

Here $\kappa_{\mathrm{f}}$ is the ratio of bulk kinetic energy to vacuum energy. And the spectral shape is given

$$
S_{\mathrm{sw}}(f)=\left(\frac{f}{f_{\mathrm{sw}}}\right)^{3}\left(\frac{7}{4+3\left(f / f_{\mathrm{sw}}\right)^{2}}\right)^{7 / 2}
$$

with approximate peak frequency

$$
f_{\mathrm{sw}}=19 \times 10^{-6} \mathrm{~Hz} \frac{1}{v_{\mathrm{w}}}\left(\frac{\beta}{H_{*}}\right)\left(\frac{T_{*}}{100 \mathrm{GeV}}\right)\left(\frac{g_{*}}{100}\right)^{\frac{1}{6}},
$$

The gravitational wave power spectrum from Kolmogorov-type turbulence is [110, 111]

$$
h^{2} \Omega_{\text {turb }}(f)=3.35 \times 10^{-4} v_{\mathrm{w}}\left(\frac{H_{*}}{\beta}\right)\left(\frac{\kappa_{\text {turb }} \alpha}{1+\alpha}\right)^{\frac{3}{2}}\left(\frac{100}{g_{*}}\right)^{\frac{1}{3}} S_{\text {turb }}(f) .
$$

Here $\kappa_{\text {turb }}$ is the efficiency of conversion of latent heat into turbulent flows. The spectral shape of the turbulent contribution is

$$
S_{\text {turb }}(f)=\frac{\left(f / f_{\text {turb }}\right)^{3}}{\left[1+\left(f / f_{\text {turb }}\right)\right]^{\frac{11}{3}}\left(1+8 \pi f / h_{*}\right)}
$$

where $h_{*}$ is the Hubble rate at $T_{*}$,

$$
h_{*}=16.5 \mu \mathrm{Hz}\left(\frac{T_{*}}{100 \mathrm{GeV}}\right)\left(\frac{g_{*}}{100}\right)^{\frac{1}{6}} .
$$

The peak frequency $f_{\text {turb }}$ is

$$
f_{\text {turb }}=27 \mu \mathrm{Hz} \frac{1}{v_{\mathrm{w}}}\left(\frac{\beta}{H_{*}}\right)\left(\frac{T_{*}}{100 \mathrm{GeV}}\right)\left(\frac{g_{*}}{100}\right)^{\frac{1}{6}} .
$$

It is a remarkable fact that four factors $\kappa_{\phi}, \kappa_{\mathrm{f}}, \kappa_{\mathrm{turb}}$ and $v_{\mathrm{w}}$ are model-dependent. If we focus on the case of Jouguet detonations [113-116], then

$$
\begin{aligned}
\kappa_{\phi} & =\frac{1}{1+0.715 \alpha}\left(0.715 \alpha+\frac{4}{27} \sqrt{\frac{3 \alpha}{2}}\right), \\
\kappa_{\mathrm{f}} & =\frac{\sqrt{\alpha}}{0.135+\sqrt{\alpha+0.98}}, \\
v_{\mathrm{w}} & =\frac{\sqrt{1 / 3}+\sqrt{\alpha^{2}+2 \alpha / 3}}{1+\alpha} .
\end{aligned}
$$

We can compute the latent heat and $\alpha$ by

$$
\begin{aligned}
\varepsilon_{*} & =\left.\left(-\Delta F(T)+T \frac{d \Delta F(T)}{d T}\right)\right|_{T=T_{*}}, \\
\alpha & =\frac{\varepsilon_{*}}{\pi^{2} g_{*} T_{*}^{4} / 30} .
\end{aligned}
$$




\begin{tabular}{|c|c|c|c|}
\hline & $\alpha$ & $H_{*} / \beta$ & $g_{*}$ \\
\hline QCD & 0.611 & $1 / 10$ & 10 \\
EW Model I $N_{\mathrm{TC}}=3$ & 6.885 & $1 / 100$ & 100 \\
EW Model I $N_{\mathrm{TC}}=4$ & 12.245 & $1 / 100$ & 100 \\
EW Model I $N_{\mathrm{TC}}=5$ & 19.136 & $1 / 100$ & 100 \\
EW Model II $N_{\mathrm{TC}}=3$ & 6.037 & $1 / 100$ & 100 \\
EW Model II $N_{\mathrm{TC}}=4$ & 10.732 & $1 / 100$ & 100 \\
EW Model II $N_{\mathrm{TC}}=5$ & 16.769 & $1 / 100$ & 100 \\
\hline
\end{tabular}

Table 2. Parameters $\alpha, H_{*} / \beta$ and $g_{*}$ used to calculate the GWs in the holographic QCD model and holographic EW model, respectively.

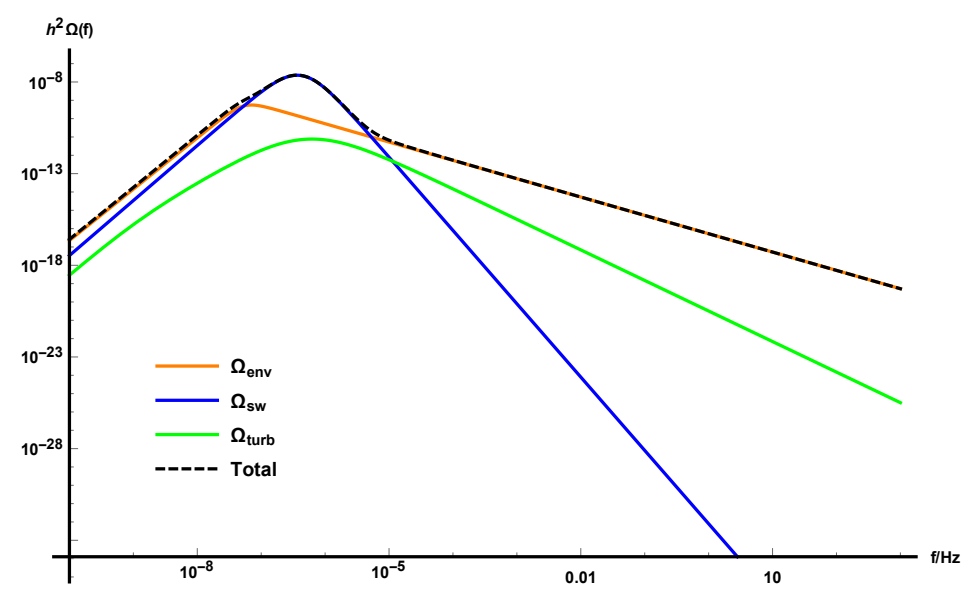

Figure 3. The GWs spectrum or the energy density of the GWs produced from the first order QCD phase transition by using the holographic QCD model.

The refs. $[8,108]$ suggest that only at most $5-10 \%$ of the bulk motion from the bubble walls is converted into vorticity, so we assume $\kappa_{\text {turb }}=0.05 \kappa_{\phi}$. Using holographic models for QCD and EW phase transitions, we can calculate the transition temperature $T_{*}$ and phase transition strength $\alpha$, and obtain the gravitational wave power spectrum.

GWs from QCD phase transition. For the dynamical holographic QCD model in section 2 , with the parameters $\mu_{G}=0.75 \mathrm{GeV}$ and $G_{5}=1.25$, the critical temperature for the first order phase transition is $T_{*}=255 \mathrm{MeV}$, and one can calculate $\alpha=0.611$. Since this holographic QCD model is for pure gluon system and quark dynamics has not been considered, we cannot obtain the specific values of $g_{*}$ and $H_{*} / \beta$. So we impose typical values of $g_{*}=10$ and $H_{*} / \beta=1 / 10$ for the QCD phase transition $[117,118]$ as listed in table 2. The gravitational wave power spectra produced from QCD phase transition is shown in figure 3 . It is found that the peak frequency is located around $3 \times 10^{-7} \mathrm{~Hz}$, and the location of the peak frequency is determined by the sound waves. The bubble collisions contribution to the GWs spectrum is dominant in the frequency region $f<5 \times 10^{-8} \mathrm{~Hz}$ and 
$f>10^{-5} \mathrm{~Hz}$, and the contribution from sound waves to the GWs spectrum is dominated in the region of $f<10^{-5} \mathrm{~Hz}$. The contribution from turbulence to the GWs spectrum is comparably small.

GWs from EW phase transition. For holographic EW model, the latent heat and $\alpha$ can be derived analytically:

$$
\begin{aligned}
\varepsilon_{*} & =\left.\left(-\Delta F(T)+T \frac{d \Delta F(T)}{d T}\right)\right|_{T=T_{*}}=\frac{N_{\mathrm{TC}}^{2} \pi^{2} T_{*}^{4}}{2}\left(2 e^{\frac{c}{\pi^{2} T_{*}^{2}}}-1\right), \\
\alpha & =\frac{\varepsilon_{*}}{\pi^{2} g_{*} T_{*}^{4} / 30}=\frac{3 N_{\mathrm{TC}}^{2}}{20}\left(2 e^{\frac{c}{\pi^{2} T_{*}^{2}}}-1\right),
\end{aligned}
$$

where we use $L^{3} / \kappa_{5}^{2}=N_{\text {TC }}^{2} /\left(4 \pi^{2}\right)$. Since the fermion part of $5 \mathrm{D}$ action has not been considered, we cannot obtain the specific values of $g_{*}$ and $H_{*} / \beta$, we impose typical values of $g_{*}=100$ and $H_{*} / \beta=1 / 100$ for EW phase transition. We estimate the value of $H_{*} / \beta$ by the nucleation of the black hole. According to [119-121], the density of fluctuations to produce a black hole of critical mass is estimated to be,

$$
n_{*}=\left(\frac{m_{p}}{\pi}\right)^{3} \exp \left(-\frac{\Delta \mathcal{F}}{T}\right)
$$

where $m_{p}$ is Planck mass and $\Delta \mathcal{F}$ is the difference between black hole and thermal gas free energy. If we consider the typical volume at phase transition is $T^{-3}$, then the free energy is $\mathcal{F}=F T^{-3}$. The rate of change of $n_{*}$ is given by

$$
\Gamma=\frac{d n_{*}}{d t}=\frac{d n_{*}}{d T} \frac{d T}{d t} .
$$

The inverse duration of the phase transition $\beta$ is

$$
\beta=\frac{1}{\Gamma} \frac{d \Gamma}{d t} .
$$

The temperature decrease rate is governed by the Hubble rate $d T / d t \simeq-T H_{*}$, so the parameter $\beta / H_{*}$ can be calculated by

$$
\frac{\beta}{H_{*}}=-\frac{T}{\Gamma} \frac{d \Gamma}{d T},
$$

where the Hubble parameter at the temperature is

$$
H_{*}=\sqrt{\frac{8 \pi^{3} g_{*}}{90}} \frac{T_{*}^{2}}{m_{p}} .
$$

According to numerical results, we can get $\beta / H_{*} \simeq 10^{2}$. In figure 4 and 5 , we plot the gravitational wave power spectra for Model I and Model II with parameters listed in table 1 of section 3 .

Our models give a strong first-order phase transition, thus the transition proceeds via detonation. Through Jouguet detonation, we can estimate the bubble velocity $v_{w}$ given by 

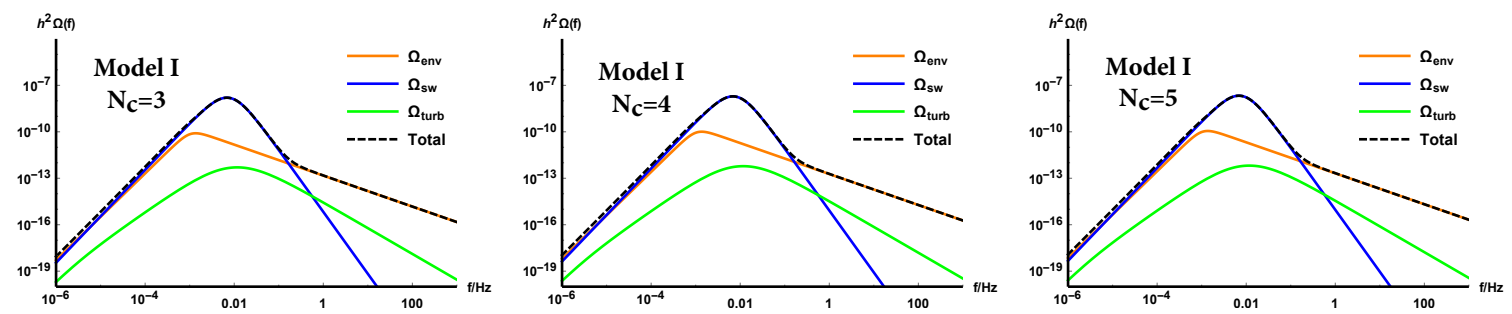

Figure 4. The GWs spectrum or the energy density of the GWs as a function of the frequency produced from the first order EW phase transition, with the parameters listed in Model I of the holographic technicolor model for $N_{\mathrm{TC}}=3, N_{\mathrm{TC}}=4$ and $N_{\mathrm{TC}}=5$, respectively.

eq. (4.15), which depends only on the ratio of vacuum energy density over radiation energy density $\alpha$. Using the parameter sets in our model, we have $\alpha \gtrsim 6$ for electroweak case, $\alpha \simeq 0.6$ for $\mathrm{QCD}$ case, which gives the bubble velocity $v_{w} \gtrsim 0.9$. According to numerical results, the higher velocity, the higher bubble collision, sound wave and turbulence GWs can be obtained. High velocity provides the necessary conditions for producing gravitational radiation.

For parameters in Model I, the critical temperature for the fist order EW phase transition is at $T_{*}=356,358,360 \mathrm{GeV}$ for $N_{T C}=3,4,5$, respectively. For parameters in Model II, the critical temperature for the fist order EW phase transition is fixed at $T_{*}=100 \mathrm{GeV}$ for $N_{T C}=3,4,5$. From figure 4 and 5 , we can see that the peak frequency is located at around $f=0.007 \mathrm{~Hz}$ for Model I and around $f=0.002 \mathrm{~Hz}$ for Model II. Similar to the QCD case, the location of the peak frequency is determined by the sound waves. The bubble collisions contribution to the GWs spectrum is dominant in the frequency region $f<10^{-6} \mathrm{~Hz}$ and $f>0.5 \mathrm{~Hz}$ for Model I and dominant in the frequency region $f<10^{-5} \mathrm{~Hz}$ and $f>0.2 \mathrm{~Hz}$ for Model II, and the contribution from sound waves to the GWs spectrum is dominated in the region of $f<0.5 \mathrm{~Hz}$ for Model I and in the region of $f<0.2 \mathrm{~Hz}$ for Model II. The contribution from turbulence to the GWs spectrum is comparably small. In the technicolor model [46], the peak frequency of the GWs produced from EW phase transition is located at around $f=0.002 \mathrm{~Hz}$, which is similar to our Model II. However, the energy density around the peak frequency region in the technicolor model [46] is around $10^{-14}-10^{-13}$, which is much weaker than that in our holographic technicolor model.

We combine the GWs spectra produced from QCD and EW phase transitions in figure 6 and show the frequency and energy density region for different GWs detectors $[122,123]$. It can be read that the energy density of GWs produced from first order QCD phase transition can reach $10^{-8}$ around the peak frequency region $3 \times 10^{-7} \mathrm{~Hz}$, which might be detected by FAST [124] and SKA, and the energy density of GWs produced from first order EW phase transition can reach $10^{-8}$ around the peak frequency $0.002 \sim 0.007 \mathrm{~Hz}$, which can be detected by BBO, DECIGO, LISA and eLISA. 

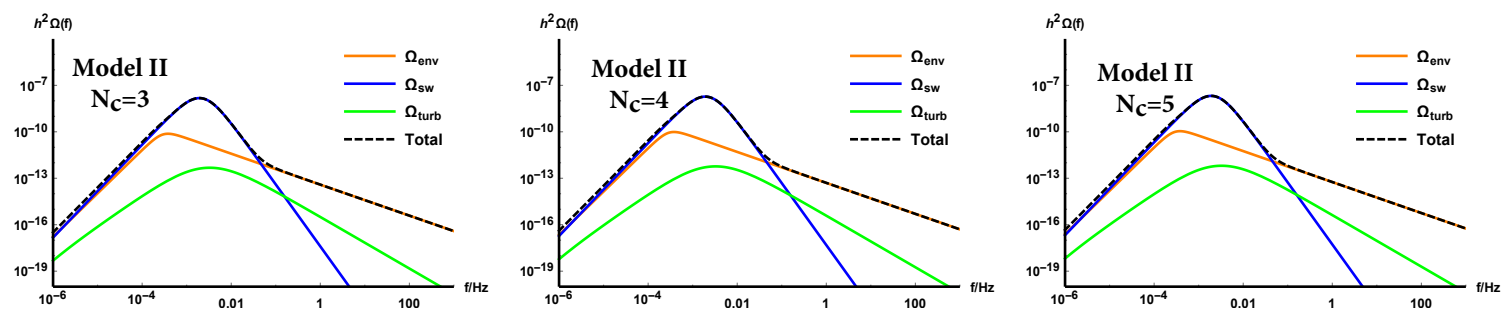

Figure 5. The GWs spectrum or the energy density of the GWs as a function of the frequency produced from the first order EW phase transition, with the parameters listed in Model II of the holographic technicolor model for $N_{\mathrm{TC}}=3, N_{\mathrm{TC}}=4$ and $N_{\mathrm{TC}}=5$, respectively.

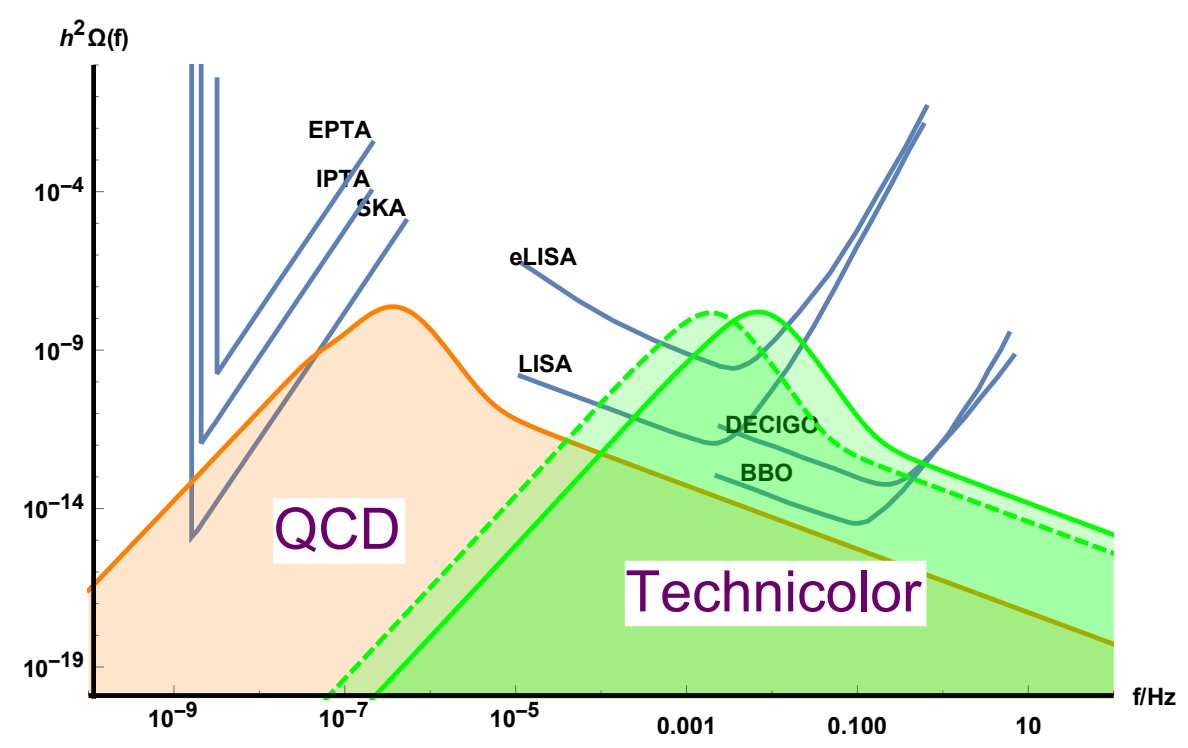

Figure 6. The combined GWs spectra from QCD and EW phase transitions and compare with possible GWs detectors.

\section{Conclusions and discussions}

In this work, we have investigated the GWs produced from QCD and EW phase transitions in the framework of holographic QCD model and holographic technicolor model.

The quenched dynamical holographic QCD model describes the pure gluon system, and the first order phase transition for confinement-deconfinement occurs at the critical temperature $255 \mathrm{MeV}$ with the parameters fixed by glueball spectra. The energy density of the GWs produced from QCD phase transition can reach $10^{-8}$ around the peak frequency region $3 \times 10^{-7} \mathrm{~Hz}$, which might be detected by the GWs detector FAST and SKA.

The holographic technicolor model is constructed by mimicking the phenomenological "Dp-Dq" system, the probe flavor " $D q$ " brane has $\mathrm{SU}\left(N_{\mathrm{TF}}\right)_{L} \times \mathrm{SU}\left(N_{\mathrm{TF}}\right)_{R}$ gauge symmetry with $N_{\mathrm{TF}}$ the number of techni-flavors living on the $\left(\mathrm{AdS}_{5}\right)$ background " $D p$ " brane. The higgs particle and techni-mesons are generated by the probe flavor brane action, and the thermodynamical properties of the system is dominated by the background brane action. For the minimal technicolor model, we have two sets of parameters corresponding to Model 
I and Model II, respectively. For Model I, by fitting free parameters $z_{m}, M, c$ from three experimental data: $S$ parameter, mass of higgs boson and vacuum expectation value of electroweak, the model can have first order EW phase transition at the critical temperature around $350 \mathrm{GeV}$. For Model II, we fix the critical temperature for EW at $100 \mathrm{GeV}$. With the critical temperature ranging from $100-360 \mathrm{GeV}$, the energy density of GWs produced from first order EW phase transition can reach $10^{-8}$ around the peak frequency $0.002 \sim 0.007$ $\mathrm{Hz}$, which can be detected by BBO, DECIGO, LISA and eLISA.

We also observe that for both GWs produced from QCD and EW phase transitions, in the peak frequency region, the dominant contribution comes from the sound waves, and away from the peak frequency region, the contribution from bubble collision is dominant to the GWs.

\section{Acknowledgments}

We thank valuable discussion with F.P. Huang, D.N. Li, Z.B. Li and Y.J.Zhao. M.Huang is supported by the NSFC under Grant No. 11725523, 11735007 and 11261130311(CRC 110 by DFG and NSFC), and Q.S. Yan is supported by the NSFC under the grant NO. 11575005 .

Open Access. This article is distributed under the terms of the Creative Commons Attribution License (CC-BY 4.0), which permits any use, distribution and reproduction in any medium, provided the original author(s) and source are credited.

\section{References}

[1] A. Einstein, Approximative integration of the field equations of gravitation, Sitzungsber. Preuss. Akad. Wiss. Berlin (Math. Phys.) 1916 (1916) 688 [inSPIRE].

[2] A. Einstein, Über Gravitationswellen (in German), Sitzungsber. Preuss. Akad. Wiss. Berlin (Math. Phys.) 1918 (1918) 154 [inSPIRE].

[3] Virgo and LIGO Scientific collaborations, B.P. Abbott et al., Observation of gravitational waves from a binary black hole merger, Phys. Rev. Lett. 116 (2016) 061102 [arXiv: 1602.03837] [INSPIRE].

[4] Virgo and LIGO Scientific collaborations, B.P. Abbott et al., GW170817: observation of gravitational waves from a binary neutron star inspiral, Phys. Rev. Lett. 119 (2017) 161101 [arXiv: 1710.05832] [INSPIRE].

[5] R.-G. Cai, Z. Cao, Z.-K. Guo, S.-J. Wang and T. Yang, The gravitational-wave physics, Natl. Sci. Rev. 4 (2017) 687 [arXiv:1703.00187] [INSPIRE].

[6] A. Kosowsky, M.S. Turner and R. Watkins, Gravitational waves from first order cosmological phase transitions, Phys. Rev. Lett. 69 (1992) 2026 [INSPIRE].

[7] A. Kosowsky and M.S. Turner, Gravitational radiation from colliding vacuum bubbles: envelope approximation to many bubble collisions, Phys. Rev. D 47 (1993) 4372 [astro-ph/9211004] [INSPIRE].

[8] C. Caprini et al., Science with the space-based interferometer eLISA. II: gravitational waves from cosmological phase transitions, JCAP 04 (2016) 001 [arXiv: 1512.06239] [INSPIRE]. 
[9] R. Jinno, K. Nakayama and M. Takimoto, Gravitational waves from the first order phase transition of the Higgs field at high energy scales, Phys. Rev. D 93 (2016) 045024 [arXiv: 1510.02697] [INSPIRE].

[10] K. Kajantie, M. Laine, K. Rummukainen and M.E. Shaposhnikov, Is there a hot electroweak phase transition at $m_{H}$ larger or equal to $m_{W}$ ?, Phys. Rev. Lett. 77 (1996) 2887 [hep-ph/9605288] [INSPIRE].

[11] M. Gurtler, E.-M. Ilgenfritz and A. Schiller, Where the electroweak phase transition ends, Phys. Rev. D 56 (1997) 3888 [hep-lat/9704013] [INSPIRE].

[12] F. Csikor, Z. Fodor and J. Heitger, Endpoint of the hot electroweak phase transition, Phys. Rev. Lett. 82 (1999) 21 [hep-ph/9809291] [INSPIRE].

[13] V. Barger, P. Langacker, M. McCaskey, M.J. Ramsey-Musolf and G. Shaughnessy, LHC phenomenology of an extended Standard Model with a real scalar singlet, Phys. Rev. D 77 (2008) 035005 [arXiv:0706.4311] [INSPIRE].

[14] S. Profumo, M.J. Ramsey-Musolf and G. Shaughnessy, Singlet Higgs phenomenology and the electroweak phase transition, JHEP 08 (2007) 010 [arXiv:0705.2425] [INSPIRE].

[15] P.H. Damgaard, A. Haarr, D. O'Connell and A. Tranberg, Effective field theory and electroweak baryogenesis in the singlet-extended Standard Model, JHEP 02 (2016) 107 [arXiv: 1512.01963] [INSPIRE].

[16] V. Vaskonen, Electroweak baryogenesis and gravitational waves from a real scalar singlet, Phys. Rev. D 95 (2017) 123515 [arXiv:1611.02073] [INSPIRE].

[17] F.P. Huang, Y. Wan, D.-G. Wang, Y.-F. Cai and X. Zhang, Hearing the echoes of electroweak baryogenesis with gravitational wave detectors, Phys. Rev. D 94 (2016) 041702 [arXiv: 1601.01640] [INSPIRE].

[18] F.P. Huang and X. Zhang, Probing the hidden gauge symmetry breaking through the phase transition gravitational waves, arXiv:1701.04338 [INSPIRE].

[19] F.P. Huang and J.-H. Yu, Explore inert dark matter blind spots with gravitational wave signatures, arXiv:1704.04201 [INSPIRE].

[20] Q.-H. Cao, F.P. Huang, K.-P. Xie and X. Zhang, Testing the electroweak phase transition in scalar extension models at lepton colliders, Chin. Phys. C 42 (2018) 023103 [arXiv: 1708.04737] [INSPIRE].

[21] F.P. Huang and C.S. Li, Probing the baryogenesis and dark matter relaxed in phase transition by gravitational waves and colliders, Phys. Rev. D 96 (2017) 095028 [arXiv: 1709.09691] [INSPIRE].

[22] A. Beniwal, M. Lewicki, J.D. Wells, M. White and A.G. Williams, Gravitational wave, collider and dark matter signals from a scalar singlet electroweak baryogenesis, JHEP 08 (2017) 108 [arXiv: 1702 .06124] [inSPIRE].

[23] C.-Y. Chen, J. Kozaczuk and I.M. Lewis, Non-resonant collider signatures of a singlet-driven electroweak phase transition, JHEP 08 (2017) 096 [arXiv:1704.05844] [INSPIRE].

[24] J.M. Cline and P.-A. Lemieux, Electroweak phase transition in two Higgs doublet models, Phys. Rev. D 55 (1997) 3873 [hep-ph/9609240] [INSPIRE]. 
[25] L. Fromme, S.J. Huber and M. Seniuch, Baryogenesis in the two-Higgs doublet model, JHEP 11 (2006) 038 [hep-ph/0605242] [INSPIRE].

[26] G.C. Dorsch, S.J. Huber and J.M. No, A strong electroweak phase transition in the 2HDM after LHC8, JHEP 10 (2013) 029 [arXiv:1305.6610] [INSPIRE].

[27] A. Haarr, A. Kvellestad and T.C. Petersen, Disfavouring electroweak baryogenesis and a hidden Higgs in a CP-violating two-Higgs-doublet model, arXiv:1611.05757 [INSPIRE].

[28] J.F. Gunion, R. Vega and J. Wudka, Higgs triplets in the Standard Model, Phys. Rev. D 42 (1990) 1673 [INSPIRE].

[29] P. Fileviez Perez, H.H. Patel, M. Ramsey-Musolf and K. Wang, Triplet scalars and dark matter at the LHC, Phys. Rev. D 79 (2009) 055024 [arXiv:0811.3957] [InSPIRE].

[30] B.M. Dillon, B.K. El-Menoufi, S.J. Huber and J.P. Manuel, A rapid holographic phase transition with brane-localized curvature, arXiv:1708.02953 [INSPIRE].

[31] S. Anand, U.K. Dey and S. Mohanty, Effects of QCD equation of state on the stochastic gravitational wave background, JCAP 03 (2017) 018 [arXiv:1701.02300] [INSPIRE].

[32] R. Jinno, S. Lee, H. Seong and M. Takimoto, Gravitational waves from first-order phase transitions: towards model separation by bubble nucleation rate, JCAP 11 (2017) 050 [arXiv: 1708.01253] [INSPIRE].

[33] R. Jinno and M. Takimoto, Gravitational waves from bubble dynamics: beyond the envelope, arXiv: 1707.03111 [INSPIRE].

[34] L. Marzola, A. Racioppi and V. Vaskonen, Phase transition and gravitational wave phenomenology of scalar conformal extensions of the Standard Model, Eur. Phys. J. C 77 (2017) 484 [arXiv: 1704.01034] [INSPIRE].

[35] W. Chao, H.-K. Guo and J. Shu, Gravitational wave signals of electroweak phase transition triggered by dark matter, JCAP 09 (2017) 009 [arXiv: 1702.02698] [INSPIRE].

[36] L. Bian, H.-K. Guo and J. Shu, Gravitational waves, baryon asymmetry of the universe and electric dipole moment in the CP-violating NMSSM, arXiv:1704.02488 [INSPIRE].

[37] W. Chao, W.-F. Cui, H.-K. Guo and J. Shu, Gravitational wave imprint of new symmetry breaking, arXiv:1707.09759 [INSPIRE].

[38] V.A. Kuzmin, V.A. Rubakov and M.E. Shaposhnikov, On the anomalous electroweak baryon number nonconservation in the early universe, Phys. Lett. B 155 (1985) 36 [INSPIRE].

[39] M.E. Shaposhnikov, Baryon asymmetry of the universe in standard electroweak theory, Nucl. Phys. B 287 (1987) 757 [InSPIRE].

[40] S. Weinberg, Implications of dynamical symmetry breaking, Phys. Rev. D 13 (1976) 974 [Addendum ibid. D 19 (1979) 1277] [INSPIRE].

[41] L. Susskind, Dynamics of spontaneous symmetry breaking in the Weinberg-Salam theory, Phys. Rev. D 20 (1979) 2619 [inSPIRE].

[42] K. Yamawaki, M. Bando and K.-I. Matumoto, Scale invariant technicolor model and a technidilaton, Phys. Rev. Lett. 56 (1986) 1335 [INSPIRE].

[43] M. Bando, K.-I. Matumoto and K. Yamawaki, Technidilaton, Phys. Lett. B 178 (1986) 308 [INSPIRE]. 
[44] M. Bando, T. Morozumi, H. So and K. Yamawaki, Discriminating technicolor theories through flavor changing neutral currents: walking or standing coupling constants?, Phys. Rev. Lett. 59 (1987) 389 [INSPIRE].

[45] J.M. Cline, M. Jarvinen and F. Sannino, The electroweak phase transition in nearly conformal technicolor, Phys. Rev. D 78 (2008) 075027 [arXiv:0808.1512] [INSPIRE].

[46] M. Jarvinen, C. Kouvaris and F. Sannino, Gravitational techniwaves, Phys. Rev. D 81 (2010) 064027 [arXiv:0911.4096] [inSPIRE].

[47] Z. Fodor and S.D. Katz, A new method to study lattice QCD at finite temperature and chemical potential, Phys. Lett. B 534 (2002) 87 [hep-lat/0104001] [INSPIRE].

[48] H.-T. Ding, F. Karsch and S. Mukherjee, Thermodynamics of strong-interaction matter from lattice QCD, Int. J. Mod. Phys. E 24 (2015) 1530007 [arXiv:1504.05274] [InSPIRE].

[49] B. Lucini, A. Rago and E. Rinaldi, $\mathrm{SU}\left(N_{c}\right)$ gauge theories at deconfinement, Phys. Lett. B 712 (2012) 279 [arXiv:1202.6684] [INSPIRE].

[50] F. Xu, H. Mao, T.K. Mukherjee and M. Huang, Dressed Polyakov loop and flavor dependent phase transitions, Phys. Rev. D 84 (2011) 074009 [arXiv:1104.0873] [INSPIRE].

[51] O. Aharony, S.S. Gubser, J.M. Maldacena, H. Ooguri and Y. Oz, Large N field theories, string theory and gravity, Phys. Rept. 323 (2000) 183 [hep-th/9905111] [INSPIRE].

[52] O. Aharony, The non AdS/non CFT correspondence, or three different paths to QCD, in Progress in string, field and particle theory: proceedings, NATO Advanced Study Institute, EC Summer School, Cargese France, 25 June-11 July 2002, pg. 3 [hep-th/0212193] [INSPIRE].

[53] A. Zaffaroni, RTN lectures on the non AdS/non CFT correspondence, PoS (RTN2005) 005 [INSPIRE].

[54] J. Erdmenger, N. Evans, I. Kirsch and E. Threlfall, Mesons in gauge/gravity duals - a review, Eur. Phys. J. A 35 (2008) 81 [arXiv:0711.4467] [inSPIRE].

[55] J.M. Maldacena, The large $N$ limit of superconformal field theories and supergravity, Int. $J$. Theor. Phys. 38 (1999) 1113 [hep-th/9711200] [INSPIRE].

[56] S.S. Gubser, I.R. Klebanov and A.M. Polyakov, Gauge theory correlators from noncritical string theory, Phys. Lett. B 428 (1998) 105 [hep-th/9802109] [INSPIRE].

[57] E. Witten, Anti-de Sitter space and holography, Adv. Theor. Math. Phys. 2 (1998) 253 [hep-th/9802150] [INSPIRE].

[58] J. Erlich, E. Katz, D.T. Son and M.A. Stephanov, QCD and a holographic model of hadrons, Phys. Rev. Lett. 95 (2005) 261602 [hep-ph/0501128] [INSPIRE].

[59] L. Da Rold and A. Pomarol, The scalar and pseudoscalar sector in a five-dimensional approach to chiral symmetry breaking, JHEP 01 (2006) 157 [hep-ph/0510268] [INSPIRE].

[60] A. Karch, E. Katz, D.T. Son and M.A. Stephanov, Linear confinement and AdS/QCD, Phys. Rev. D 74 (2006) 015005 [hep-ph/0602229] [INSPIRE].

[61] T. Sakai and S. Sugimoto, Low energy hadron physics in holographic QCD, Prog. Theor. Phys. 113 (2005) 843 [hep-th/0412141] [INSPIRE].

[62] T. Sakai and S. Sugimoto, More on a holographic dual of QCD, Prog. Theor. Phys. 114 (2005) 1083 [hep-th/0507073] [INSPIRE]. 
[63] G.F. de Teramond and S.J. Brodsky, Hadronic spectrum of a holographic dual of QCD, Phys. Rev. Lett. 94 (2005) 201601 [hep-th/0501022] [INSPIRE].

[64] L. Da Rold and A. Pomarol, Chiral symmetry breaking from five dimensional spaces, Nucl. Phys. B 721 (2005) 79 [hep-ph/0501218] [INSPIRE].

[65] K. Ghoroku, N. Maru, M. Tachibana and M. Yahiro, Holographic model for hadrons in deformed AdS $S_{5}$ background, Phys. Lett. B 633 (2006) 602 [hep-ph/0510334] [INSPIRE].

[66] O. Andreev and V.I. Zakharov, Gluon condensate, Wilson loops and gauge/string duality, Phys. Rev. D 76 (2007) 047705 [hep-ph/0703010] [INSPIRE].

[67] M. Kruczenski, L.A. Pando Zayas, J. Sonnenschein and D. Vaman, Regge trajectories for mesons in the holographic dual of large- $N_{c}$ QCD, JHEP 06 (2005) 046 [hep-th/0410035] [INSPIRE].

[68] H. Forkel, M. Beyer and T. Frederico, Linear square-mass trajectories of radially and orbitally excited hadrons in holographic QCD, JHEP 07 (2007) 077 [arXiv:0705.1857] [INSPIRE].

[69] Y. Chen and M. Huang, Two-gluon and trigluon glueballs from dynamical holography QCD, Chin. Phys. C 40 (2016) 123101 [arXiv:1511.07018] [InSPIRE].

[70] A. Ballon-Bayona, H. Boschi-Filho, L.A.H. Mamani, A.S. Miranda and V.T. Zanchin, Effective holographic models for QCD: glueball spectrum and trace anomaly, Phys. Rev. D 97 (2018) 046001 [arXiv: 1708.08968] [INSPIRE].

[71] O. DeWolfe, S.S. Gubser and C. Rosen, A holographic critical point, Phys. Rev. D 83 (2011) 086005 [arXiv: 1012.1864] [INSPIRE].

[72] O. DeWolfe, S.S. Gubser and C. Rosen, Dynamic critical phenomena at a holographic critical point, Phys. Rev. D 84 (2011) 126014 [arXiv:1108.2029] [INSPIRE].

[73] Y. Yang and P.-H. Yuan, A refined holographic QCD model and QCD phase structure, JHEP 11 (2014) 149 [arXiv:1406.1865] [INSPIRE].

[74] R. Critelli, J. Noronha, J. Noronha-Hostler, I. Portillo, C. Ratti and R. Rougemont, Critical point in the phase diagram of primordial quark-gluon matter from black hole physics, Phys. Rev. D 96 (2017) 096026 [arXiv: 1706.00455] [INSPIRE].

[75] Z. Li, Y. Chen, D. Li and M. Huang, Locating the QCD critical end point through the peaked baryon number susceptibilities along the freeze-out line, Chin. Phys. C 42 (2018) 013103 [arXiv: 1706.02238] [INSPIRE].

[76] K. Haba, S. Matsuzaki and K. Yamawaki, Holographic techni-dilaton, Phys. Rev. D 82 (2010) 055007 [arXiv: 1006 .2526] [INSPIRE].

[77] S. Matsuzaki and K. Yamawaki, Holographic techni-dilaton at 125 GeV, Phys. Rev. D 86 (2012) 115004 [arXiv:1209.2017] [INSPIRE].

[78] D.K. Hong and H.-U. Yee, Holographic estimate of oblique corrections for technicolor, Phys. Rev. D 74 (2006) 015011 [hep-ph/0602177] [INSPIRE].

[79] J. Hirn and V. Sanz, A negative S parameter from holographic technicolor, Phys. Rev. Lett. 97 (2006) 121803 [hep-ph/0606086] [INSPIRE].

[80] M. Piai, Precision electro-weak parameters from $A d S_{5}$, localized kinetic terms and anomalous dimensions, hep-ph/0608241 [INSPIRE]. 
[81] C.D. Carone, J. Erlich and J.A. Tan, Holographic bosonic technicolor, Phys. Rev. D 75 (2007) 075005 [hep-ph/0612242] [INSPIRE].

[82] C. Núñez, I. Papadimitriou and M. Piai, Walking dynamics from string duals, Int. J. Mod. Phys. A 25 (2010) 2837 [arXiv:0812.3655] [InSPIRE].

[83] L. Anguelova, P. Suranyi and L.C.R. Wijewardhana, Holographic walking technicolor from D-branes, Nucl. Phys. B 852 (2011) 39 [arXiv:1105.4185] [INSPIRE].

[84] L. Anguelova, P. Suranyi and L.C.R. Wijewardhana, Scalar mesons in holographic walking technicolor, Nucl. Phys. B 862 (2012) 671 [arXiv:1203.1968] [INSPIRE].

[85] D. Elander and M. Piai, The decay constant of the holographic techni-dilaton and the $125 \mathrm{GeV}$ boson, Nucl. Phys. B 867 (2013) 779 [arXiv:1208.0546] [INSPIRE].

[86] R. Contino, Y. Nomura and A. Pomarol, Higgs as a holographic pseudo-Goldstone boson, Nucl. Phys. B 671 (2003) 148 [hep-ph/0306259] [InSPIRE].

[87] K. Agashe, R. Contino and A. Pomarol, The minimal composite Higgs model, Nucl. Phys. B 719 (2005) 165 [hep-ph/0412089] [INSPIRE].

[88] K. Agashe and R. Contino, The minimal composite Higgs model and electroweak precision tests, Nucl. Phys. B 742 (2006) 59 [hep-ph/0510164] [INSPIRE].

[89] D. Croon, B.M. Dillon, S.J. Huber and V. Sanz, Exploring holographic composite Higgs models, JHEP 07 (2016) 072 [arXiv: 1510.08482] [INSPIRE].

[90] D. Espriu and A. Katanaeva, Holographic description of $\mathrm{SO}(5) \rightarrow \mathrm{SO}(4)$ composite Higgs model, arXiv: 1706.02651 [INSPIRE].

[91] A. Adams, L.D. Carr, T. Schäfer, P. Steinberg and J.E. Thomas, Strongly correlated quantum fluids: ultracold quantum gases, quantum chromodynamic plasmas and holographic duality, New J. Phys. 14 (2012) 115009 [arXiv:1205.5180] [INSPIRE].

[92] O. Andreev and V.I. Zakharov, Heavy-quark potentials and AdS/QCD, Phys. Rev. D 74 (2006) 025023 [hep-ph/0604204] [INSPIRE].

[93] H.J. Pirner and B. Galow, Strong equivalence of the AdS-metric and the QCD running coupling, Phys. Lett. B 679 (2009) 51 [arXiv:0903.2701] [INSPIRE].

[94] J. Erlich, E. Katz, D.T. Son and M.A. Stephanov, QCD and a holographic model of hadrons, Phys. Rev. Lett. 95 (2005) 261602 [hep-ph/0501128] [INSPIRE].

[95] P. Colangelo, F. De Fazio, F. Giannuzzi, F. Jugeau and S. Nicotri, Light scalar mesons in the soft-wall model of AdS/QCD, Phys. Rev. D 78 (2008) 055009 [arXiv:0807.1054] [INSPIRE].

[96] T. Gherghetta, J.I. Kapusta and T.M. Kelley, Chiral symmetry breaking in the soft-wall AdS/QCD model, Phys. Rev. D 79 (2009) 076003 [arXiv:0902.1998] [InSPIRE].

[97] Y.-Q. Sui, Y.-L. Wu, Z.-F. Xie and Y.-B. Yang, Prediction for the mass spectra of resonance mesons in the soft-wall AdS/QCD with a modified $5 D$ metric, Phys. Rev. D 81 (2010) 014024 [arXiv:0909.3887] [inSPIRE].

[98] Y.-Q. Sui, Y.-L. Wu and Y.-B. Yang, Predictive AdS/QCD model for mass spectra of mesons with three flavors, Phys. Rev. D 83 (2011) 065030 [arXiv: 1012.3518] [INSPIRE].

[99] D. Li, M. Huang and Q.-S. Yan, A dynamical soft-wall holographic QCD model for chiral symmetry breaking and linear confinement, Eur. Phys. J. C 73 (2013) 2615

[arXiv: 1206.2824] [INSPIRE]. 
[100] D. Li and M. Huang, Dynamical holographic QCD model for glueball and light meson spectra, JHEP 11 (2013) 088 [arXiv:1303.6929] [INSPIRE].

[101] D. Li, S. He, M. Huang and Q.-S. Yan, Thermodynamics of deformed AdS $S_{5}$ model with a positive/negative quadratic correction in graviton-dilaton system, JHEP 09 (2011) 041 [arXiv:1103.5389] [INSPIRE].

[102] D. Li, J. Liao and M. Huang, Enhancement of jet quenching around phase transition: result from the dynamical holographic model, Phys. Rev. D 89 (2014) 126006 [arXiv:1401.2035] [INSPIRE].

[103] D. Li, S. He and M. Huang, Temperature dependent transport coefficients in a dynamical holographic QCD model, JHEP 06 (2015) 046 [arXiv:1411.5332] [INSPIRE].

[104] Y. Chen and M. Huang, Two-gluon and trigluon glueballs from dynamical holography QCD, Chin. Phys. C 40 (2016) 123101 [arXiv:1511.07018] [INSPIRE].

[105] Particle Data Group collaboration, C. Patrignani et al., Review of particle physics, Chin. Phys. C 40 (2016) 100001 [InSPIRE].

[106] S.J. Huber and T. Konstandin, Gravitational wave production by collisions: more bubbles, JCAP 09 (2008) 022 [arXiv:0806.1828] [INSPIRE].

[107] R. Jinno and M. Takimoto, Gravitational waves from bubble collisions: an analytic derivation, Phys. Rev. D 95 (2017) 024009 [arXiv: 1605.01403] [INSPIRE].

[108] M. Hindmarsh, S.J. Huber, K. Rummukainen and D.J. Weir, Numerical simulations of acoustically generated gravitational waves at a first order phase transition, Phys. Rev. D 92 (2015) 123009 [arXiv: 1504.03291] [INSPIRE].

[109] M. Hindmarsh, S.J. Huber, K. Rummukainen and D.J. Weir, Shape of the acoustic gravitational wave power spectrum from a first order phase transition, Phys. Rev. D 96 (2017) 103520 [arXiv: 1704.05871] [inSPIRE].

[110] C. Caprini, R. Durrer and G. Servant, The stochastic gravitational wave background from turbulence and magnetic fields generated by a first-order phase transition, JCAP 12 (2009) 024 [arXiv:0909.0622] [INSPIRE].

[111] P. Binetruy, A. Bohe, C. Caprini and J.-F. Dufaux, Cosmological backgrounds of gravitational waves and eLISA/NGO: phase transitions, cosmic strings and other sources, JCAP 06 (2012) 027 [arXiv: 1201.0983] [InSPIRE].

[112] D.J. Weir, Gravitational waves from a first order electroweak phase transition: a brief review, Phil. Trans. Roy. Soc. Lond. A 376 (2018) 20170126 [arXiv:1705. 01783] [INSPIRE].

[113] P.J. Steinhardt, Relativistic detonation waves and bubble growth in false vacuum decay, Phys. Rev. D 25 (1982) 2074 [inSPIRE].

[114] M. Kamionkowski, A. Kosowsky and M.S. Turner, Gravitational radiation from first order phase transitions, Phys. Rev. D 49 (1994) 2837 [astro-ph/9310044] [INSPIRE].

[115] A. Nicolis, Relic gravitational waves from colliding bubbles and cosmic turbulence, Class. Quant. Grav. 21 (2004) L27 [gr-qc/0303084] [INSPIRE].

[116] J.R. Espinosa, T. Konstandin, J.M. No and G. Servant, Energy budget of cosmological first-order phase transitions, JCAP 06 (2010) 028 [arXiv: 1004.4187] [INSPIRE]. 
[117] M. Ahmadvand and K. Bitaghsir Fadafan, Gravitational waves generated from the cosmological QCD phase transition within AdS/QCD, Phys. Lett. B 772 (2017) 747 [arXiv: 1703.02801] [INSPIRE].

[118] M. Ahmadvand and K. Bitaghsir Fadafan, The cosmic QCD phase transition with dense matter and its gravitational waves from holography, Phys. Lett. B 779 (2018) 1 [arXiv: 1707.05068] [INSPIRE].

[119] D.J. Gross, M.J. Perry and L.G. Yaffe, Instability of flat space at finite temperature, Phys. Rev. D 25 (1982) 330 [INSPIRE].

[120] J.I. Kapusta, Nucleation rate for black holes, Phys. Rev. D 30 (1984) 831 [INSPIRE].

[121] J.I. Kapusta and C. Gale, Finite-temperature field theory: principles and applications, Cambridge University Press, Cambridge U.K., (2011) [INSPIRE].

[122] Gravitational wave detectors and sources webpage, http://rhcole.com/apps/GWplotter/.

[123] K. Kuroda, W.-T. Ni and W.-P. Pan, Gravitational waves: classification, methods of detection, sensitivities and sources, Int. J. Mod. Phys. D 24 (2015) 1530031 [arXiv: 1511.00231] [INSPIRE].

[124] R. Nan et al., The Five-hundred-meter Aperture Spherical Radio Telescope (FAST) project, Int. J. Mod. Phys. D 20 (2011) 989 [arXiv:1105.3794] [INSPIRE]. 